\title{
INTERACTIVE METHODS OF TRAINING IN SUSTAINABLE WASTE MANAGEMENT
}

\author{
Bogdan Nikolov ${ }^{1}$, Slaveya Petrova $^{2}$, Vanya Naydenova $^{3}$, Delka Karagyozova-Dilkova ${ }^{4}$
}

\begin{abstract}
Environmental education addresses the most common and current environmental problems as they could lead to a serious ecological misbalance on Earth. One of them is associated with a huge amount of waste, the need for sustainable management and recovery. For this reason, the topic of sustainable waste management needs to be included in the process of environmental education, to be based on an interdisciplinary approach, to start from an early age and be embedded not only in school curricula but also in extra-curricular activities, to be conducted systematically and methodologically provided.

This paper presents an interactive model for training in sustainable waste management through an activity approach that could be successfully applied in some extra-curricular activities. Didactic instruments have been developed in the course of a project funded by the University of Plovdiv, aiming to enhance the ecological competencies of both pupils (11-13 years' age) and students (pre-teachers in Biology). The thematic content included four themes: Waste definition and classification, Waste treatment, the Recycling process, and the Composting process. Learning activities and the expected results of the training are defined for each theme. The innovative model of training in sustainable waste management includes four of the most popular interactive educational methods: research-based education, problem-based education, game-based education, and project learning. Worksheets have been developed for all activities, and the thematic content was structured following environmental topics, enabling environmental education through a proactive approach. In order to assess the effectiveness of the interactive methodology, two questionnaires (pre-test and post-test) were developed. Each one consisted of 15 questions allowing the analysis of the environmental competencies of pupils (knowledge, attitude and behavior construct), as well as the attitudes, habits and behavior of both children and their families to the problem of sustainable waste management. The first questionnaire was made before the training in order to determine the entry-level of knowledge and competencies, while the second one was conducted after the training in order to evaluate the competencies' enhancement.

The experimental trainings conducted (2019-2020) show that these models for training in sustainable waste management, based on the constructivist approach, lead to a significant increase of pro-ecological behavior and can be successfully implemented in the education process. According to the knowledge construct, we found that the working hypothesis was confirmed for $80.2 \%$ of the pupils $\left(\chi^{2}=2.043, \mathrm{p}<0.05\right)$. Based on the attitude construct, we found that the working hypothesis was confirmed for $46.7 \%$ of the pupils $\left(\chi^{2}=1.205, \mathrm{p}<0.05\right)$. In the behavior construct, the working hypothesis was confirmed for almost $23 \%$ of the pupils who had not had long-term habits for nature conservation and rational use of resources before the time of training $\left(\chi^{2}=0.383, \mathrm{p}<0.05\right)$. This was the construct with the lowest positive change from the three studied.
\end{abstract}

UDC Classification: 37.04, DOI: https://doi.org/10.12955/pns.v2.157

Keywords: sustainable development, recycle-reuse-reduce, separate waste collection, pro-ecological behavior.

\section{Introduction}

Environmental education is a priority and occupies a key place in the education system. It is most often interpreted as a psychological and pedagogical process aimed at mastering a system of knowledge, skills and habits. In the process of environmental education, students acquire knowledge about the most common and current environmental problems, as they can be a serious threat to the ecological balance. One of them is associated with a huge amount of waste, the need for sustainable waste management and recovery. For this reason, the topic of sustainable waste management needs to be included in the process of ecological education, even at the earliest stage.

The effectiveness of learning depends on many factors, one of which is the student's involvement in learning activities. Traditional educational practice is based on the premise that knowledge is something that can be passed directly from the teacher to the students. Passive, reading and writing oriented pedagogy with traditional content and organization is accepted as the norm.

Constructivism differs from the traditional notion that knowledge exists independently of the individual and is based on the premise that man actively constructs his own understanding or knowledge. Based on his experience, he builds his own understanding of the world in which he lives - chooses and transforms information, builds hypotheses and makes decisions.

\footnotetext{
${ }^{1}$ Plovdiv University „Paisii Hilendarski“, Faculty of Biology, Department of Ecology and Environmental Conservation, Plovdiv, Bulgaria, nikolov81bg@yahoo.com

2 Plovdiv University „Paisii Hilendarski“, Faculty of Biology, Department of Ecology and Environmental Conservation, Plovdiv, Bulgaria, sl.petrova@abv.bg

${ }^{3}$ Plovdiv University - „Lyuben Karavelov“ Branch, Department of Pedagogy, Kardzhali, Bulgaria, vanianay@abv.bg

${ }^{4}$ Plovdiv University „Paisii Hilendarski“, Faculty of Biology, Department of Botany and Teaching Methods in Biology, Plovdiv, Bulgaria, dkarag@abv.bg
} 
This paper presents some interactive methods of training in sustainable waste management: researchbased education, problem-based education, game-based education, and project learning.

Our hypothesis: if the training in sustainable waste management is carried out through an activity approach, the environmental culture and environmental competence of pupils and students will be enhanced, and a long-lasting pro-environmental behavior will be formed. Two questionnaires (pre-test and post-test) were developed in order to account for the effectiveness of the innovative methodological model by measuring the three constructs - knowledge, attitude and behavior.

\section{Literature review}

Modern theoretical formulations of psychology, pedagogy and didactics provide an opportunity to build a methodological model based on the theory of constructivism (Karagyozova-Dilkova, 2007).

The constructivist theory is well-known for more than 80 years and is widely explained and developed in the scientific works of Dewey (1938), Piaget (1953), Vygotsky (1978), and Bruner (1985). The main idea is that learners (students) are actively involved in the learning process and in the construction of their own knowledge based on their experience. This idea is very well explained by Woolfolk (1993) "learning is active mental work, not passive reception of teaching".

Some of the main elements of the theory of constructivist learning are as follows (Kurt, 2021):

- Knowledge is constructed - Everyone starts their learning with some preexisting knowledge and upgrades on it. That's why everyone's knowledge is unique.

- Learning is a social activity - The approach of constructivism includes different interactions between students (group work, discussions, etc.) in order to reflect on each one's past experience.

- Learning is an active process - All learners (students) are actively involved in many projects, games, quizzes, discussions in order to explore a problem, to obtain new data, and finally to construct the knowledge.

- Learning is contextual - Learning occurs in the context of our own lives, based on our own experience. According to the constructivist theory, we have to reflect on ourselves and classify the new data obtained from our current perspective.

- Learning exists in the mind - The active engagement of students in the learning process and a reflection on their own experience are key factors for knowledge construction.

- Knowledge is personal - As everyone has a unique perspective and their own experience, so will be the knowledge gained.

- Motivation is the key to learning - The active engagement of learners (students) in different types of planned activities is due to the educator's skills to motivate and to enhance their interest.

The above-mentioned concepts of constructivism clearly indicate the great opportunities and advantages concerning the formation of key competencies in adolescents, pupils and students. For this reason, we have decided to develop our innovative model for training in sustainable waste management based on the activity approach.

\section{Data and methodology}

Interactive technology has been developed for the study of sustainable waste management through an activity approach (Nikolov et al., 2020). Didactic instruments have been developed in the course of a project funded by the University of Plovdiv aiming to enhance the ecological competencies of both pupils (11-13 years' age) and students (pre-teachers in Biology).

The thematic content included four themes: Waste definition and classification, Waste treatment, the Recycling process, and the Composting process. Learning activities and the expected results of the training are defined for each theme. Worksheets have been developed for all activities, and the thematic content was structured following environmental topics, enabling environmental education through a proactive approach (Nikolov et al., 2020).

The innovative model of training in sustainable waste management includes four of the most popular interactive educational methods: research-based education, problem-based education, game-based education, and project learning. The process of cognitive activity takes place in the cycle of D. Kolb (1984) and is based on the previous experience of the learners. This methodological model is based on 
activity learning, including certain tasks that are performed in small groups (about 4-5 students). After solving the tasks, the results are communicated to the whole class and discussed between the students and the teacher, with the teacher having a leading role. The tasks set for the study of the basic concepts are divided into 4 stages and arranged into a system, which is based on the learning cycle of D. Kolb (experience, reflection, conceptualization of experience, and the application of acquired experience). Students work independently within the group and research and construct knowledge and ideas. They have an active role in the learning process and do not passively receive information from the teacher, which determines the interactive and innovative nature of the learning process.

In order to assess the effectiveness of the interactive methodology, two questionnaires (pre-test and posttest) were developed. Each questionnaire consisted of 15 questions allowing the analysis of the environmental competencies of pupils (knowledge, attitude, and the behavior construct), as well as the attitudes, habits and behavior of both children and their families to the problem of sustainable waste management. The first questionnaire was conducted before the training in order to determine the entrylevel of knowledge and competencies, while the second one was conducted after the training in order to evaluate the competencies' enhancement.

More than 300 pupils of the 11-13 years' age and 15 Bachelors students (pre-teachers on Biology) from the Faculty of Biology at the Plovdiv University "Paisii Hilendarski" (Plovdiv, Bulgaria) participated in the training in sustainable waste management. The results obtained from the two questionnaires were subjected to mathematical and statistical analysis to verify their reliability using the statistical software SPSS vers. 12. The following methods were used - descriptive statistics, test for significant differences between samples (t-test) and testing of the working hypothesis $\left(\chi^{2}\right)$ at $\mathrm{p}<0.05$.

\section{Results and Discussion}

\section{Inquiry and Problem-based learning}

Basic inquiry learning was firstly described by John Dewey (1938). This approach starts with the teacher presenting some problems that the students have to address by gathering information and testing their conclusions. According to Woolfolk (2007), this approach includes some student activities as follows: 1) formulation of hypotheses; 2) data collection; 3 ) evaluation of data; 4) reflection on the problem and proposing solutions.

This method is applied in the training of the first two themes - "Waste definition and classification" and "Waste treatment". In the beginning, the teacher asks everyone to write on a sheet of paper what kind and amount of waste he has thrown away that day. The list of waste is then summarized, and it is concluded that every day each of us disposes of many different types of waste.

The next step is to divide the class into groups where each group is working with a specific type of waste - household, construction, industrial, medical, agricultural waste, etc. For example, the first group works with household waste, the second group with medical waste, and so on. Each group must tell what they do with this type of waste in their daily lives, whether these actions are right or not, and if they are not right, how we can improve our actions and deeds in order to protect the natural environment. Finally, after all the groups have presented their views, the teacher summarizes that the amount of waste generated in the modern world is huge and that it must be managed in a sustainable way to avoid a number of environmental problems.

\section{Research-based and Project-based education}

The approach of project-based learning is characterized by a problem that has to be explored in order to find a solution. Students explore the problem by doing research to find some missing information. The next step is to formulate some hypotheses and propose some possible solutions. Then, students apply their new knowledge, evaluate the solutions, and reflect on the knowledge and skills they have gained (Woolfolk, 2007). These methods could be successfully applied in the training of almost all themes of sustainable waste management.

For example, the activities in "Waste treatment" could start by asking the pupils to present their personal observations and impressions - "After we dispose of various waste, they go to the landfill, but what happens to them there?", "What can we do with the accumulated waste in the landfill?", "What can we do to prevent negative consequences on the environment?". Pupils answer the questions, express their opinions, and share ideas for addressing the problem. The next step is for group work, where pupils 
should do research on waste treatment processes and technologies. Finally, each one concludes that the conversion of a product into waste is not the final stage of the waste life cycle, that the waste "lives" in different environments for a long time and so they can cause serious environmental problems in nature. When regarding the themes "Recycling process" and "Composting process", the project-based education method is very suitable. The class is divided into several groups, and each one has to do a project on the main stages of the processes - composting of biodegradable waste, recycling of plastics, glass, paper, metal, respectively. Each group works independently, having to complete the task for a certain time. Once everyone is ready, each group presents their work to the whole class and explains the different stages of recycling or composting of waste. At the end of the lesson, students enrich their knowledge about the advantages of the recycling and composting process by addressing sustainable waste management. They come to the conclusion on their own: each one must be engaged in protecting the environment by creating habits for the separate collection and disposal of waste, which will facilitate and shorten the process of their recovery.

\section{Game-based education}

Learning, in the process of a game, is strongly emotionally colored and aims to help students in the organization of knowledge, so we decided to create an interesting game - Let recycle together!

Students are divided equally into 2 or 3 teams. Each team must sit together in a small group so that they can consult with each other for the correct answers. The first team to gather in one place and choose a captain starts first.

The game was developed as a PowerPoint presentation (Fig. 1), contains 20 questions, divided into 4 categories - "Is it recyclable?", "How to recycle?", "The recycling process", "Recycling facts". Each category contains 5 questions with different levels of difficulty, carrying from 10 to 50 points, respectively. The presentation starts in "Slideshow" mode, and the participants are instructed about the rules of the game.

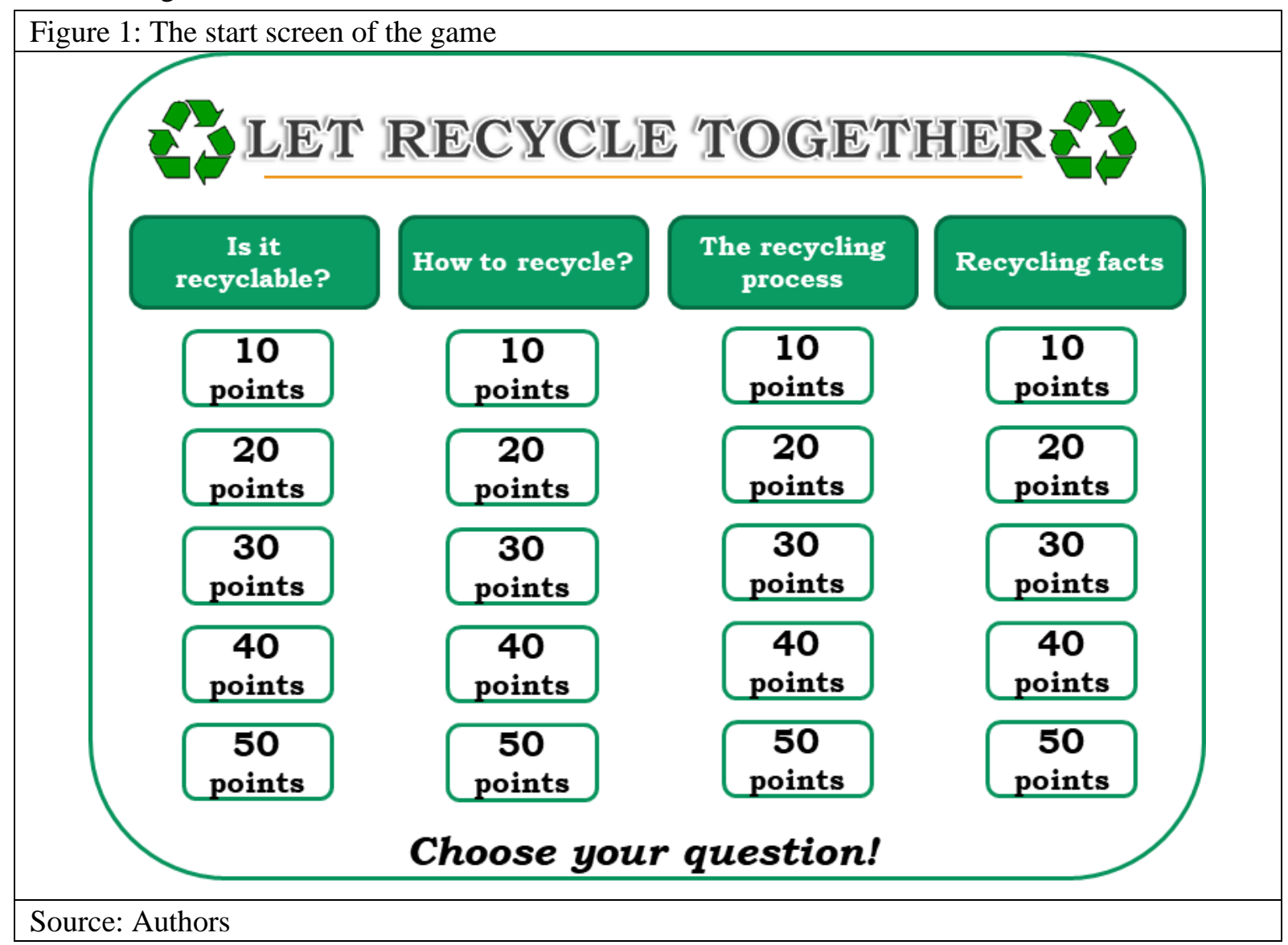

The first team chooses a section and the number of points per question from the given section. Press the appropriate number on the scoreboard to display the question and time to receive an answer (30 seconds or 1 minute). After receiving a response from the team, press once more to display the correct answer. 
If the team has answered correctly, the points indicated for the respective question are recorded in their favor.

Then we return to the scoreboard and continue the game until the questions are exhausted. Some questions have the option of a bonus question. Each bonus question allows all teams to answer additional questions for 10 points each. The winner is the team with the highest score.

Assessment of the effectiveness of the interactive methods of training in sustainable waste management

Two questionnaires (pre-test and post-test) were used in the assessment of the effectiveness of the innovative methodological model for training in sustainable waste management. Their questions were designed in such a way that they allow analyzing the ecological competencies (knowledge construct), the attitudes (attitude construct), and behavior (behavior construct) of the pupils (Vassileva, 2013).

The knowledge construct was evaluated based on the answers to the following questions/propositions:

1) "Why do we collect waste separately?" - "Because everyone does", "To save resources and energy", "I have no idea."

2) "Before we throw plastics in the colored waste container, we must ..." - explain the steps you make to prepare this waste for disposal

3) "Before we throw paper in the colored waste container, we must ..." - explain the steps you make to prepare this waste for disposal

4) "Before we throw glass in the colored waste container, we must ..." - explain the steps you make to prepare this waste for disposal

5) "Which group of waste is the most dangerous for nature?" - "paper", "plastics", "glass", "metal", "food."

6) "Connect each of the proposed waste with the respective colored container."

7) "How do you assess your own knowledge on issues related to environmental protection?" 1 =insufficient, $2=$ little, $3=$ =medium, $4=$ =sufficient, $5=$ =very in-depth

The attitude construct was evaluated based on the answers to the following questions/propositions:

1) "I collect all my waste paper, plastic, and glass separately " - "yes", "no."

2) "I throw my waste separately because ..." - "I want to protect nature", "My families do this at home", "All my friends do this."

3) "I do not throw out my waste separately because ..." - "This is pointless", "We do not have separate bins at home", "None of my friends does this."

4) "We repair some items at home instead of throwing them away" - "yes", "mostly", "no."

5) "I prefer to avoid and use stuff with disposable packages" - "yes", "no."

The Behavior construct was evaluated based on the answers to the following questions/propositions:

1) "My waste today could fit in ..." - "small box", "small bin", "big bin."

2) "I repeatedly use items rather than throw them away ..." - "yes", "mostly", "no."

3) "What type of shopping bag are you using in the supermarket?" - "plastic bag", "canvas bag", "paper bag."

All raw data from the pre-test and post-test answers were mathematically processed in order to find any differences and tendencies as a result of the training in sustainable waste management. We found a statistically significant increase in the level of knowledge of both pupils and students, according to the post-test answers $(\mathrm{p}<0.05)$. Significant knowledge enhancement was observed with regard to the correct behavior and activities (steps) for the disposal of plastic and paper waste in colored containers (by 33\%) ( $\mathrm{p}<0.05$ ), and with regard to glass, there is a slight increase (by $8 \%$ ), due to the high input level results. As a result of the training about sustainable waste management, we reported an increase in the share of pupils and their families who have already started collecting waste separately, but at a lower significance level $(\mathrm{p}<0.01)$.

According to the knowledge construct, we found that the working hypothesis was confirmed for $80.2 \%$ of the pupils $\left(\chi^{2}=2.043, \mathrm{p}<0.05\right)$. Based on the attitude construct, we found that the working hypothesis was confirmed for $46.7 \%$ of the pupils $\left(\chi^{2}=1.205, \mathrm{p}<0.05\right)$. Based on the behavior construct, the working hypothesis was confirmed for almost $23 \%$ of the pupils who had not had long-term habits for nature conservation and the rational use of resources before the training $\left(\chi^{2}=0.383, p<0.05\right)$. This was the construct with the lowest positive change from the three studied. 


\section{Conclusion}

The experimental trainings conducted in 2019-2020 show that these models for training in sustainable waste management, based on the constructivist approach, lead to a significant increase of pro-ecological behavior and can be successfully implemented in the education process.

The data shows that the training in sustainable waste management has very high efficiency, and the students have significantly increased their environmental competencies, reaching a level of $72-100 \%$ according to the knowledge construct. The other two constructs are affected to a lesser extent, which correlates with data from surveys conducted among households at the national level. This fact emphasizes the need to direct the training on sustainable waste management to adolescents because there is the strongest increase in environmental competencies and lasting habits of pro-environmental attitudes and behavior.

In conclusion, we can recommend the developed methodical model for training in sustainable waste management through an activity approach to be implemented in all school grades. The model is open to being supplemented and enriched with initiatives and campaigns planned at the local and national levels. This will enable students to be actively involved in social life, in addressing community problems and subsequently, this will lead to the formation of sustainable environmental behavior, which is essential for nature conservation for both current and future generations.

\section{Acknowledgements}

This research work was carried out with the support of the Scientific Fund at the Plovdiv University „Paisii Hilendarski“ by contract SP19BF008, managed by Assoc. Prof. Delka Karagyozova-Dilkova.

\section{References}

Bruner, J. (1985). Vygotsky: A Historical and Conceptual Perspective. In J. V. Wertsch (Ed.), Culture, Communication and Cognition: Vygotskian Perspectives (pp. 21-35). Cambridge: Cambrige University Press.

Dewey. J. (1938). Experience and Education. New York: Collier Books.

Karagyozova-Dilkova, D. (2007). Personality-oriented strategies in Biology education. Plovdiv

Killermann, W. (1998). Research into biology teaching methods. Journal of Biological Education, 33(1): 4-9

Kolb, D. A. (1984). Experiential learning: Experience as the source of learning and development (Vol. 1). Englewood Cliffs, NJ, Prentice-Hall.

Kurt, S. (2021). Constructivist Learning Theory, in Educational Technology, February 21, 2021. Retrieved from https://educationaltechnology.net/constructivist-learning-theory/

Nikolov, B., Stankova, G., Tabak, A., Kateva, M., Shamburova, R., Dzhambazova, P., Petrova, S., Naydenova, V.,

Karagyozova-Dilkova, D. (2020). Methodological model for enhancement of the ecological competences of students through sustainable waste management. Proceedings of CBU International Conference on Innovations in Science and Education, 2022 March 2020, Prague, Czech Republic, in press

Piaget, J. (1953). Origins of Intelligence in the Child. London: Routledge and Kegan Paul.

Vygotsky, L. (1978). Mind and Society: The Development of Higher Psychological Processes. Cambridge, Massatchusetts: Harvard University Press.

Woolfolk, A. E. (1993). Educational psychology. Boston: Allyn and Bacon.

Woolfolk, A. (2007). Social cognitive and constructivist views of learning (Chapter 9). Educational Psychology (p. 204-245). Upper Saddle River, NJ: Prentice Hall. 